\title{
Stopping flies in their tracks
}

\begin{abstract}
A new red-shifted anion channelrhodopsin brings fast optical inhibition to the Drosophila field.
\end{abstract}

"In Drosophila people have been manipulating circuits for close to 18 years", says Adam Claridge-Chang, Assistant Professor at Duke-NUS medical school in Singapore. But the field, according to Claridge-Chang, has been missing, until recently, crucial tools in wide use in other model organisms. Perhaps most conspicuously missing from the field's arsenal is channelrhodopsin, arguably the most popular optogenetic tool used by neuroscientists for activating neural circuits.

There's a bit of irony in this absence, given that the concept of 'optogenetics' and its first in vivo demonstration to control neural activity and animal behavior was performed in flies by Gero Miesenbock's group, where Claridge-Chang previously trained as a postdoc.

The problem, Claridge-Chang explains, is that commonly used channelrhodopsin channels require blue light for activation, and the Drosophila cuticle is actually opaque to blue light, leaving channelrhodopsin relatively useless to many neuroscientists in the fly community. This problem was solved around 2013, when two red-shifted channelrhodopsins were described, allowing stimulation with light that easily penetrates the fly cuticle.

The second important missing tool has been a good optogenetic inhibitor. Halorhodopsin, a popular light-activated silencer of neural activity, is also difficult to apply in Drosophila. Now, in a recent Nature Methods paper (doi:10.1038/ nmeth.4148; published online 23 January 2017), Claridge-Chang's lab overcomes this hurdle for rapid inhibition of fly neural activity, by applying a newly discovered line of algal anion channelrhodopsins, known as Guillardia theta anion channelrhodopsins (GtACRs). GtACRs have several attractive features as a neural inhibitor for fly researchers, including activation by green light, which easily penetrates Drosophila for rapid silencing of neurons in vivo. Using physiology and a variety of behavioral assays, the paper demonstrates GtACR's ability to rapidly silence targeted neurons and drive immediate behavioral changes.

Claridge-Chang hopes this new tool for rapidly silencing the fly nervous system will enable some qualitatively new experiments, like real-time dynamic feedback experiments where the animal's initiation of a behavior can trigger light pulses and neural inactivation. But beyond technical improvements, he hopes better tools for neural silencing will shift researchers away from the perspective of "activationism." "Neuroscientists tend to think about quiet circuits and the effects of activating them. But brains are not quiet, even at rest. By allowing us to impose quiescence on neurons, we can start looking at what happens when normally active circuits are silenced." Dustin M. Graham 